\title{
Fundamentación de la visita domiciliaria desde un enfoque de enfermería familiar y cultural
}

\author{
Foundation of home visits from a family and culture nursing perspective
}

Fundamentação da visita domiciliar desde o enfoque de enfermagem familiar e cultural

\author{
Alexandra Giraldo-Osorio* \\ Diana Paola Betancurth-Loaiza** \\ Liliana Orozco-Castillo ${ }^{* * *}$ \\ Adriana Milena Mejía-Alarcón ${ }^{* * * *}$
}

\section{Resumen}

Introducción: La visita domiciliaria permite el abordaje de la salud desde un enfoque biopsicosocial y de determinantes sociales. El objetivo fue analizar la evidencia científica sobre las teorías, modelos y enfoques para la enfermería familiar y cultural que permitan la fundamentación de la visita domiciliaria. Metodología: Se realizó una investigación documental entre 2007 y 2017 en inglés, español y portugués; sobre bases de datos de salud y de ciencias sociales y humanas. Los términos utilizados fueron: teoría de enfermería, visita domiciliaria, cuidado de enfermería, cultura, familia. Se hallaron 671 artículos científicos. Para el análisis se utilizó el software Atlas ti. Resultados: El concepto de visita domiciliaria se configura desde una visión plural, se destaca la inclusión de una dimensión política y se plantea la necesidad de trascender el predominante modelo médico hegemónico y proponer la visita desde un abordaje comprensivo y cultural. Conclusiones: Se identificaron teorías/modelos de enfermería y de psicología utilizados en el abordaje de la visita domiciliaria desde un enfoque familiar y cultural. Prima el modelo biomédico, por lo que se plantean enfoques alternativos para la praxis y es relevante profundizar sobre los postulados de la teoría de la diversidad y universalidad: cuidados culturales propuesta por Madeleine Leininger.

Palabras clave: Visita domiciliaria, cuidados de enfermería, enfermería en salud comunitaria, teoría de enfermería, atención primaria de salud.

\footnotetext{
Abstract

Introduction: Home visits allow to address health form a biopsychosocial approach and social determinants. The objective of the study consisted of analyzing scientific evidence of the family and cultural nursing theories, models and approaches that allow the foundation of home visits. Methodology: A documentary research was made between 2007 and 2017, in English, Spanish and Portuguese, in data bases of health, and social and human sciences. The terms used were: nursing theory, home visits, nursing care, culture and family. 671 scientific articles were found. For the
}

Autor de correspondencia

* Magíster en Salud Pública. Docente tiempo completo. Departamento de Salud Pública, Grupo Promoción de la Salud y Prevención de la Enfermedad, Grupo Cuidado de la Salud y la Vida Humana-CUSAVI, Universidad de Caldas. Correo: alexandra. giraldo@ucaldas.edu.co. Orcid: https:/orcid. org/0000-0003-1330-2713. Caldas, Colombia.

** Ph.D en Salud Pública. Docente tiempo completo. Departamento de Salud Pública, Grupo Cuidado de la Salud y la Vida HumanaCUSAVI, Universidad de Caldas. Correo: diana.betancurth@ucaldas.edu.co. Orcid: https://orcid.org/0000-0001-7620-2336. Caldas, Colombia.

*** Ph.D en Enfermería. Docente tiempo completo. Departamento de Salud Pública, Grupo Cuidado de la Salud y la Vida HumanaCUSAVI, Universidad de Caldas. Correo: liliana.orozco@ucaldas.edu.co. Orcid: https:// orcid.org/0000-0002-8686-6168. Caldas, Colombia.

**** Estudiante de Enfermería. Universidad de Caldas. Correo: adriana.241126050@ ucaldas.edu.co. Orcid: https://orcid.org/00000003-3998-7431. Caldas, Colombia.

(C) Universidad Francisco de Paula Santander. Este es un artículo bajo la licencia CC BY (https://creativecommons.org/ licenses/by/4.0/) @) (1) 
ISSN-PRINT

1794-9831

E-ISSN 2322-7028

Vol. 17 No. 1

Ene - Abr 2020

Cúcuta, Colombia

analysis the software Atlas ti was used. Results: The concept of home visit is configured from a plural vision which emphasizes the inclusion of political measures and states the necessity of transcending the predominant hegemonic medical model and propose home visits from a comprehensive and cultural approach. Conclusions: Nursing and psychology theories/models were identified, which were used for addressing home visits from a family and cultural approach. The biomedical model prevails, therefore alternative approaches are considered for praxis and its relevant to go in depth on the diversity and universality theory; transcultural nursing, a proposal made by Madeleine Leininger

Keywords: Home visit, nursing care, community health nursing, nursing theory, primary health care.

\begin{abstract}
Resumo
Introdução: A visita domiciliar permite a abordagem da saúde desde o enfoque biológico, psicológico e de determinantes sociais. O objetivo do estudo consistiu em analisar a evidência científica sobre teorias, modelos e enfoques para a enfermagem familiar e cultural que permitam a fundamentação da visita domiciliar. Metodologia: Realizou-se uma pesquisa documental entre 2007 e 2017, em inglês, espanhol e português, empregando buscadores da área da saúde, ciências sociais e humanas. Os descritores empregador foram: teoria de enfermagem, visita domiciliar, cuidado de enfermagem, cultura e família. Acharam-se 671 artigos científicos. Para a análise utilizou-se o software Atlas ti. Resultados: O conceito visita domiciliar configura-se desde uma visão plural, destaca-se a inclusão de uma dimensão política e planteasse a necessidade de transcender do predominante modelo médico hegemônico e propor uma visita desde a abordagem compreensiva e cultural. Conclusões: identificaram-se teorias/modelos de enfermagem e de psicologia utilizados na abordagem da visita domiciliar desde o enfoque familiar e cultural. Prima o modelo biomédico, pelo que se propõe enfoques alternativos para a práxis, sendo relevante aprofundar sobre a teoria da diversidade e a universalidade: cuidados culturais, proposta por Madeleine Leininger
\end{abstract}

Palavras chave: Visita domiciliar, cuidados de enfermagem, enfermeira em saúde comunitaria, teoria de enfermagem, atenção primária à saúde.

\section{Introducción}

La visita domiciliaria (VD) se considera un medio para fomentar el cuidado y la educación para la salud $\mathrm{y}$, de esta forma, fortalecer en las personas, familias y comunidades, la capacidad para avanzar hacia una salud más satisfactoria para ellas, según las oportunidades que tengan en sus contextos.

La Declaración de Astaná "Desde Alma-Ata hacia la cobertura Sanitaria Universal y los Objetivos de Desarrollo Sostenible", propone que el éxito de la Atención Primaria de Salud (APS) depende entre otros de (1) "utilizar los conocimientos, tanto científicos como tradicionales, para fortalecerla, mejorar los resultados de salud y garantizar el acceso de todas las personas a la atención adecuada en el momento oportuno y en el nivel de atención más apropiado, respetando sus derechos, necesidades, dignidad y autonomía".

Según Harzheim et al. (2), la academia articulada a la estrategia de APS ha tenido en cuenta a la familia, la comunidad y el entorno sociocultural como sustrato valioso para la formación del talento humano en salud; es así como se ha reconocido la importancia de estos escenarios para su formación a nivel de pregrado, posgrado y educación continuada, lo que conlleva el reto de la reorganización de las instituciones de educación superior y del sistema de salud (2-5). Además, es de resaltar que la vivienda de las personas/familias se presenta como un escenario en el cual es posible brindar un cuidado singular, humanizado y centrado en la familia y en su cultura (5); escenario en el cual se requiere la puesta en marcha de proyectos de investigación desde la disciplina de Enfermería con el fin de fortalecer su fundamentación epistemológica con relación al trabajo extramural mediado por la aplicación de los conocimientos disciplinares.

En Colombia, las VD que realiza la enfermería se han caracterizado por un fuerte enfoque biomédico dirigido a identificar factores de riesgo, al control de las enfermedades, a evitar reingresos hospitalarios y a realizar demanda inducida para dar cumplimiento a las actividades, procedimientos e intervenciones de detección temprana y protección específica, establecidas por el Ministerio de Salud y Protección Social en las Normas Técnicas o en las Guías de Práctica Clínica $(6,7)$. 
Pese a la mirada reduccionista, la práctica formativa en el marco de la VD ha evidenciado que en los estudiantes despierta el espíritu investigativo y crítico, permite el desarrollo de capacidades comunicativas, sensibiliza el compromiso social y amplía la capacidad para aportar conocimiento a las múltiples miradas de una misma realidad $(8,9)$. En ese sentido, se configura como una estrategia didáctica que permite el abordaje de la salud desde un enfoque biopsicosocial y del entorno, teniendo en cuenta la identificación y análisis de los determinantes sociales de la salud.

En enfermería, el cuidado desde un enfoque cultural permite conocer la trama de significados que los sujetos construyen $(8,10)$, el cual está atravesado por el conocimiento cotidiano que se gesta en las experiencias de vida de los sujetos, del que hacen parte también las representaciones sociales del conocimiento biomédico.

Por otra parte, la Política de Atención Integral en Salud en Colombia (11) insta a brindar una prestación de atención integral y a su vez desarrollar y fortalecer competencias para el abordaje de familias y comunidades en la modalidad extramural. Es así como el enfoque familiar y comunitario entiende que la atención de salud debe ser (11) un proceso continuo que se centra en el cuidado de las familias, se preocupa por la salud de las personas antes que aparezca la enfermedad y les entrega herramientas para el autocuidado, la gestión y la promoción de su salud.

En el caso de la enfermería no se ha establecido una teoría disciplinar que permita el desarrollo de las VD desde los enfoques planteados (familiar y cultural), pese a que, por una parte, el Ministerio de Salud y Protección Social, a través del Modelo Integral de Atención en Salud, del año 2016, hizo un llamado para el fortalecimiento de la salud pública por medio del ejercicio profesional en el marco de la estrategia de APS (11); por otra parte, se ha identificado la falta de antecedentes en la disciplina de la enfermería que presenten de manera explícita y como resultado de la investigación, las recomendaciones en cuanto a la aplicación de uno y otro enfoque, modelo o teoría en el marco de la VD, lo que ha promovido la búsqueda de estrategias que permitan dar respuesta desde la academia a esta necesidad. Por esto, el objetivo de este estudio fue analizar la evidencia científica sobre las teorías, modelos y enfoques que están relacionados con la enfermería familiar y cultural, que permitan la fundamentación de la visita domiciliaria.

\section{Metodología}

Se realizó una investigación documental, dirigida a recuperar registros sobre los conceptos de cultura, familia, visita domiciliaria, teorías sobre la enfermería y metaparadigma enfermero, con el fin de hacer un análisis reflexivo y proponer la teoría y/o modelo de enfermería que aporte al fortalecimiento del desarrollo y a la práctica de la visita que realiza el profesional de la enfermería. Como unidad de análisis se tomaron artículos científicos (de investigación, revisión y reflexión), tesis de grado y de posgrado, libros de modelos y teoristas sobre enfermería publicados entre el periodo 2007 y 2017 en español, inglés y portugués.

Como fuentes de información se utilizaron bases de datos especializadas en el área de salud y de ciencias sociales y humanas (Biblioteca Virtual en Salud, PubMed, Embase y Redalyc), también el motor de búsqueda Google Académico. A partir de los tesauros DeCs (Descriptores en Ciencias de la Salud) y MeSH (Medical Subject Headings) se estructuraron estrategias de búsqueda con los términos: teoría de enfermería, visita domiciliaria, cuidado de enfermería, cultura, familia, nursing theory, house calls, home visit, visiting nursing service, nursing care, culture, cultural anthropology y family. Se establecieron como criterios de exclusión documentos de literatura gris y que no se obtuvieran en texto completo, estudios que en el título o resumen no incluyeron los temas de la revisión y aquellos que hicieron referencia a temas relacionados, pero no directamente vinculados. En total se hallaron 671 artículos/documentos científicos (Figura 1). 
ISSN-PRINT

1794-9831

E-ISSN 2322-7028

Vol. 17 No. 1

Ene - Abr 2020

Cúcuta, Colombia

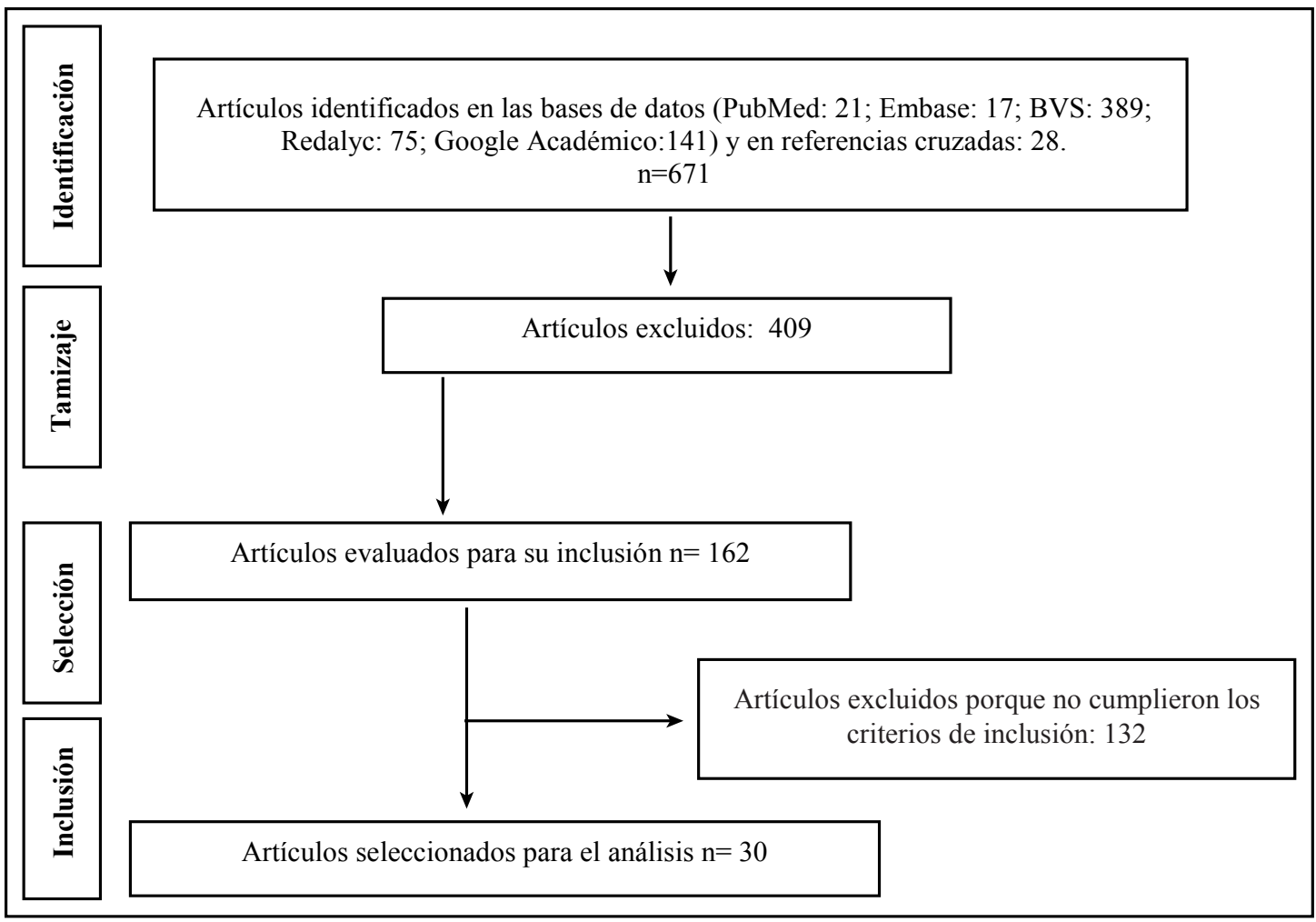

Figura 1. Diagrama de flujo de la selección de estudios a través de las diferentes fases de la investigación documental

Fuente: Elaboración propia.

Para la sistematización de la información se construyó y diligenció una base de datos en Excel con las variables de autor(es), título, resumen, año de publicación, país de realización del estudio, idioma, tipo de fuente de consulta, nombre de la teorista y denominación del enfoque propuesto; esto para la selección inicial de los registros que cumplieran con los criterios de inclusión. Posteriormente, se revisaron los textos completos, los cuales correspondieron a 30 artículos (Figura 1) por medio del software Atlas Ti Versión 8.3.1 licenciado por la Universidad de Caldas. Se codificó la información de acuerdo con las categorías preliminares que dieron pie a otras emergentes. Para el análisis se construyeron resúmenes que posibilitaron la selección de información con los aspectos más relevantes, el ordenamiento de ideas (semejanzas y diferencias), el relacionamiento lógico y la comparación constante para dar paso a la interpretación de modo que se pudiera trascender los datos fácticos $(12,13)$.
La investigación marco titulada "Enfoque de enfermería familiar y cultural para la visita domiciliaria: aporte para la atención primaria de salud", en la que se desarrolla la investigación documental fue aprobada por el Comité de Bioética de la Facultad de Ciencias para la Salud y financiada por parte de la Vicerrectoría de Investigaciones y Posgrados de la Universidad de Caldas (código 0406818).

\section{Análisis}

Al revisar los 30 artículos se encontró que Brasil es el país en el que más se han realizado estudios relacionados con VD que efectúa enfermería con un $26,7 \%$, seguido de Chile y Estados Unidos con un $10 \%$; el año de mayor publicación fue el 2011 con un 16,7\% seguido de los años 2008 y 2015 con un 13,33\% cada uno; se publicó principalmente en inglés con un 43,33\%, seguido por portugués (30\%) y español (27\%). Los tipos de estudio se relacionan a continuación en la Tabla 1. 
Tabla 1: Número de artículos según el tipo de estudio

\begin{tabular}{lcc}
\hline \multicolumn{1}{r}{ Tipo de estudio } & Frecuencia absoluta & Frecuencia relativa (\%) \\
\hline Revisión & 4 & 13,3 \\
Reflexión & 3 & 10 \\
Investigación Cuantitativa & & \\
-Ensayo controlado aleatorio & 3 & 26,66 \\
-Cohorte retrospectiva & 1 & \\
-Diseño de casos y controles & 1 & \\
-Diseño cuasi-experimental & 3 & 50 \\
Investigación Cualitativa & & \\
-Descriptiva & 11 & 100 \\
-Etnográfica, etnoenfermería & 1 & \\
-Estudio de caso & 2 & \\
-Teoría fundamentada & 1 & \\
Total & 30 & \\
\hline
\end{tabular}

E-ISSN 2322-7028

Vol. 17 No. 1

Ene - Abr 2020

Cúcuta, Colombia

Fuente: elaboración propia.

A continuación, se presentan los resultados estructurados en tres componentes que subyacen de las categorías analizadas: el concepto de visita desde una visión plural, la visita desde un enfoque biomédico y la visita desde un enfoque teórico.

\section{El concepto de visita desde una visión plural}

Se encontraron diferentes denominaciones para referirse a la visita que realizan los profesionales de enfermería y el equipo de salud en general, estas fueron: visita domiciliaria, visita familiar, visita al hogar, visita al hogar familiar, visita familiar domiciliaria, visita comunitaria, atención domiciliaria y vigilancia domiciliar. La mayoría de los artículos no sustentan teóricamente y de forma explícita las razones para referirse a la visita de una manera u otra, e incluso se encontró que, en un mismo estudio, se utilizan diferentes denominaciones de manera indiscriminada; no obstante, prima la acepción de visita domiciliaria en 12 de los 30 artículos revisados.

En cuanto a la conceptualización, se identificó a la VD como estrategia, instrumento, servicio, herramienta, programa, conjunto de acciones sistematizadas, e incluso, como una dimensión política y asistencial. Como estrategia, según Cordero y Guevara (14) se ha concebido como la principal, al acercar los servicios de salud a los domicilios y lugares de trabajo de las personas, lo cual permite conocer de primera mano las necesidades de la población, y generar beneficios tanto a nivel individual como colectivo; además, Brugerolles et al. (15), han manifestado que posibilita el desarrollo de acciones con el objetivo de promover la salud, mejorar las capacidades funcionales y prevenir la institucionalización.
Se evidencia que cuando se habla de instrumento se refiere a una intervención fundamental en la salud de la familia y en la continuidad de cualquier forma de asistencia y/o atención domiciliaria $(16,17)$, dado que da lugar a la implementación de prácticas que extrapolan el ámbito individual, para, de acuerdo con Cordero y Guevara (14), incidir en los determinantes sociales del colectivo, que incluye atender las diferentes necesidades de salud, preocupándose, entre otras, por la infraestructura existente en las comunidades. Desde una mirada crítica, Sivalli et al. (18) conciben la VD como "un instrumento de práctica protocolar, hegemónicamente realizada en los moldes de la educación higienista, apuntada en la literatura como una práctica coercitiva y controladora". Otros autores como Santos y Alves (19) la asocian con el modelo prusiano alemán de control, en que el poder que viene de esferas centrales del Estado, extrapola la privacidad de las familias.-

De acuerdo con Cordero y Guevara (14), la VD como servicio garantiza los principios filosóficos de la seguridad social, universalidad, solidaridad, igualdad y equidad. Según estos mismos autores, vista como herramienta constituye una oportunidad para implementar acciones preventivas, curativas y de promoción de la salud a los individuos en su contexto familiar, al permitir una mayor aproximación del personal de salud con la realidad y dinámica de las familias; de igual forma, es óptima ya que sirve para orientar, informar, educar y establecer alianzas para el empoderamiento y promoción de la autonomía en la salud. Como programa es entendida comouna modalidad para el apoyo a la familia en el primer nivel de atención, a través de encuentros periódicos proporcionando 
ISSN-PRINT

1794-9831

E-ISSN 2322-7028

Vol. 17 No. 1

Ene - Abr 2020

Cúcuta, Colombia una amplia gama de servicios como información, guía y soporte emocional, entre otros (14).

Adicionalmente, Cordero y Guevara han descrito que (14) "no toda visita al domicilio puede ser considerada una VD, ya que para serlo debe comprender un conjunto de acciones sistematizadas que se inician antes y continúan después de la visita, además que precisa de un recurso humano con una capacitación específica". Salgueiro et al. (20) destacan también, la ocurrencia de la VD como una dimensión política y asistencial de la promoción en salud, la cual interfiere en la lógica de la oferta y demanda, por lo que la integración de la atención, la satisfacción del usuario, así como la democratización y la politización de conocimientos relacionados al proceso salud-enfermedad, actúan de manera concreta en la organización y producción de los servicios de salud.

\section{La visita desde un modelo médico hegemónico}

En cuanto a las experiencias en investigación relacionadas con las visitas domiciliarias, en los artículos encontrados predomina la realización de investigaciones en VD de enfermería que tienen que ver con los cuidados prenatales y de menores (16,21-28). Según Mikton et al. (24), la VD de la primera infancia es el tipo de intervención más evaluada con una fuerte evidencia de su efectividad para la prevención del maltrato infantil (26).

Se debe precisar, que en las visitas con enfoque prenatal, el personal de enfermería ayuda a las madres a desarrollar una relación segura con sus hijos $(16,23,24)$, trabaja en el adecuado desarrollo infantil $(16,17,22,23)$ y evalúa el estado fisiológico de la madre y del recién nacido. Por otro lado, las VD enfocadas a los menores se centran en mejorar la salud física, la mental, el bienestar cognitivo, lo social y lo emocional $(22,24)$; también, proporcionan un ambiente seguro $(22,25)$ y dan orientación en redes familiares y en los apoyos comunitarios $(21,22,24,25)$.

En cuanto al abordaje de las VD se evidenció principalmente el enfoque morbicéntrico, reflejado en actividades de la enfermería centradas en el control de medicamentos $(25,29,30)$, curaciones, vacunación y toma de muestras de laboratorio $(14,29)$; igualmente, se identificaron los riesgos sexuales y reproductivos $(23,26,31)$, el cuidado de las personas con enfermedades físicas y mentales $(14,18,26,32)$ y la derivación a otros servicios o programas $(32,33)$, con el objetivo de reducir la discapacidad, la enfermedad y la muerte prematura (16,34). Algunos estudios se ocupan de factores diferentes a la enfermedad que afectan la dinámica familiar, como son: la estructu- ra física del domicilio $(14,29)$, la educación en salud familiar (higiene, alimentación y actividad física) $(14,29)$, pero, ante todo, se resalta el carácter humanizado al darle valor a la escucha activa $(14,29) \mathrm{y} / \mathrm{o}$ la posibilidad de crear vínculos a partir de los determinantes sociales de la salud (17).

Por otra parte, los hallazgos describen otra experiencia de la VD con un enfoque cultural $(22,35)$, donde se hace referencia a la incorporación de materiales, símbolos, ideas, costumbres, creencias, valores, significados, actitudes, pensamientos y estilos de vida al contemplar un entorno interesante para explicar los patrones de salud-enfermedad de los miembros que la conforman $(35,36)$.

De acuerdo a estas experiencias de la VD, predomina el enfoque morbicéntrico, reflejado en actividades de enfermería centradas en la prevención de la enfermedad, su seguimiento, tratamiento $(14,18,26,32)$ y la derivación a otros servicios $(32,33)$, con el objetivo de reducir la discapacidad y muerte prematura (33). En contraste con lo anterior, Machado et al. (17) han destacado que, aunque las acciones curativas y de prevención de complicaciones son relevalentes, es necesario centrarse en acciones de promoción de la salud y de prevención de la enfermedad, ya que ambas hacen parte del núcleo de los servicios de salud para la atención básica $(17,37)$.

Con relación al modelo biomédico dominante en las VD, este ha permitido avances significativos en el conocimiento y desarrollo tecnológico; no obstante, al respecto Depaux et al. describieron que pierde validez científica cuando deja por fuera las variables humanas (38), lo que requiere de un cambio de paradigma. Thomas Kunh planteaba la importancia de la reorientación de la ciencia que era precedida por la "crisis" y termina en una "revolución científica"; para el caso de la atención en salud, se precisa pasar de una perspectiva biomédica a una que incluya la salud integral familiar, lo cual implica una postura reflexiva (31). Según Parra (31), este modelo se ha agotado para dar explicaciones a diferentes fenómenos, por lo cual resulta insuficiente para el equipo de salud y el profesional de la enfermería, dado que se basa en una concepción patologista y reduccionista; visión contraria a lo que se propone desde el paradigma biopsicosocial el cual se enfoca en la persona, en sus necesidades y en su contexto histórico y social; pero que además brinda orientación familiar, reivindica el sujeto y permite una mayor participación de la comunidad y la continuidad de los cuidados $(39,40)$. 
$\mathrm{A} 1$ respecto, en un estudio publicado por Brugerolles et al., en el 2008 se encontró que (15) el modelo biomédico imperante en las visitas, a pesar de superar prácticas dirigidas a aspectos disfuncionales del cuerpo biopsíquico y fenómenos característicos de las fases del ciclo vital, se desarrolla con prácticas pautadas por acciones programáticas y orientadas a factores de riesgo en grupos prioritarios (16-18); es por eso que coherentemente, las prácticas de los enfermeros han concentrado su accionar en aspectos clínicos del individuo $(14,16,17,20)$.

Por otro lado, Sivalli et al., (18) fueron críticos de la VD basada en el modelo biomédico, al referirse a ésta como una práctica controladora. Sumado a ello, Santos y Alves (19) profundizan su crítica al plantear que el personal de enfermería lleva a cabo actividades orientadas a la instrumentalización de la población, para el cuidado en la dimensión individual y colectiva.

Por lo descrito, se requiere la reflexión sobre la importancia que para la enfermería tiene la VD en el marco de la Atención Primaria de Salud, y la necesidad de problematizar las tensiones y desafíos en cuanto a la teoría y a la praxis según la evidencia encontrada.

\section{La visita desde un enfoque teórico}

Se encontraron diferentes propuestas de enfoques, teorías y modelos según las diversas escuelas del pensamiento social que orientan la VD, lo que da cuenta de la posible diversificación de esta práctica.
Desde un enfoque hermenéutico-dialéctico se hallaron algunos elementos para considerar la VD desde un enfoque comprensivo, el cual reconoce la dimensión histórico-social de los sujetos y las familias. Entre algunos elementos importantes de la VD, Alves y Acioli destacan (29) el habla, el testimonio y los textos (aquellos que se plasman en diarios de campo), como resultado de un proceso social y de conocimiento expresado en el lenguaje, ambos, producto de múltiples determinantes de la vida diaria.

Por otro lado, desde el enfoque de la corriente crítica, se encontraron estudios que basan la experiencia de la visita guiada teóricamente por el post-estructuralismo feminista $(21,41)$ y las prácticas emancipatorias $(18)$. Dentro de los argumentos expuestos se percibió la necesidad de identificar con los sujetos y sus familias, las fortalezas y la toma de decisiones en un proceso de co-construcción continuo, la ruptura de tendencias opresivas hacia las mujeres y las relaciones de poder $(21,41)$.

Con respecto a algunas experiencias éstas encontraron terreno fértil para realizar la VD en las teorias y modelos psicológicos. Desde este punto de vista, autores estadounidenses se inclinaron por teorías que promueven el cambio conductual desde el plano individual, interpersonal y comunitario $(22,23,30,42)$; mientras que en Chile, según Parra Giordano (30), su abordaje se enfocó en el modelo bio-psicosocial. Y, finalmente, Dawley y Loch (23), describen otra teoría como la de la ecología humana de Bronfenbrenner, la cual abarca desde la persona hasta el macrosistema (ver Tabla 2).

Tabla 2. Teorías y modelos de psicología utilizados en el abordaje de la visita domiciliaria.

\begin{tabular}{|c|c|c|c|c|}
\hline Teoría/modelo & Autor & Año & Característica & Referencia \\
\hline Modelo transteórico del cambio & Prochaska & 1979 & \multirow{3}{*}{ Promueven el cambio individual } & 23 \\
\hline Modelo biopsicosocial & Engel & 1977 & & 31 \\
\hline Teoría de la autoeficacia & Bandura & 1977 & & 23,32 \\
\hline Teoría del apego & Bowlby & 1969 & \multirow{2}{*}{ Promueven el cambio interpersonal } & 23 \\
\hline The Family Partnership Model & Davis & 1980 & & 22 \\
\hline $\begin{array}{l}\text { The Vulnerable Populations } \\
\text { Conceptual Model }\end{array}$ & Flaskerud \& Winslow & 1988 & Promueven el cambio comunitario o social & 42 \\
\hline Teoría de la ecología humana & Bronfenbrenner & 1979 & $\begin{array}{l}\text { Promueve cuatro esferas (microsistema, mesosistema, } \\
\text { exosistema, macrosistema) }\end{array}$ & 23 \\
\hline
\end{tabular}

Fuente: Elaboración propia 
ISSN-PRINT

1794-9831

E-ISSN 2322-7028

Vol. 17 No. 1

Ene - Abr 2020

Cúcuta, Colombia
También, se identificaron las teorías y modelos de enfermería con el fin de explicar distintos fenómenos de interés durante el proceso de la visita domiciliaria $(15$, 30,33,37,43-46) (ver Tabla 3). Los elementos que de allí subyacen permiten un conocimiento más profundo, así como develar la reflexión de su utilidad y contribución a la práctica disciplinar. En estos estudios predomina el cuidado como constructo central de la enfermería al que se le vinculan el componente cultural, la promoción de la salud, la prevención de la enfermedad y el humanismo, destacándose la estrecha relación y el diálogo entre la persona-enfermero/a.

Tabla 3. Teorías y modelos de enfermería utilizados en el abordaje de la visita domiciliaria.

\begin{tabular}{|c|c|c|c|}
\hline Teoría/modelo & Autor & Año & Referencia \\
\hline Modelo de atención enfermería & Yura y Walsh & 1967 & 33 \\
\hline Modelo de cuidados & Virginia Henderson & 1955 & 15 \\
\hline Modelo de adaptación & Callista Roy & 1976 & 44 \\
\hline Teoría de Autocuidado & Dorothea Orem & 1971 & 15,31 \\
\hline Teoría de enfermería de la universalidad y la diversidad del cuidado cultural & Madeleine Leininger & 1978 & 37,45 \\
\hline Teoría de Enfermería Humanística & Paterson y Zderad & 1976 & 43 \\
\hline Teoría del cuidado humano & Jean Watson & 1979 & 46 \\
\hline Patrones funcionales de salud & Marjory Gordon & 1970 & 30 \\
\hline
\end{tabular}

Fuente: Elaboración propia

\section{La visita desde un abordaje comprensivo y cul- tural}

Superar el marcado enfoque biomédico de la visita exige en principio dar una pelea lingüística por el uso semántico del concepto, por lo que el análisis de las diferentes acepciones de la visita en los estudios analizados muestra que se soslayan sus bases teóricas y epistemológicas. En este sentido, resulta trascendental pasar de un concepto de "visita domiciliaria" que alude a un escenario instrumental según Sivalli et al., (18) al concepto de "visita familiar", un término que de manera particular se vincula estrechamente con las personas en un sentido más humano.

El contexto familiar es un ambiente grupal que comparte lazos de parentesco o vínculos, que interactúan y se ven influenciados por factores socioculturales que determinan y transmiten costumbres, creencias, valores, significados, actitudes, pensamientos y modos de vida; por tanto, es un entorno interesante que sirve para comprender y explicar las diferentes visiones de mundo y los significados que los integrantes le atribuyen, no solo a las experiencias en relación con la enfermedad sino con la vida misma $(37,47)$.

En este caso la visita familiar se convierte en un escenario que permite confrontar el imaginario de los equipos de APS con la realidad de las familias, se- gún lo propone Granda (48) en el marco de una salud pública alternativa, lo que implica necesariamente un replanteamiento del tradicional abordaje centrado en el riesgo y en la objetivización de las personas, para "mirar e interpretar el proceso salud-enfermedad de manera distinta, es decir, ver la salud poblacional en su realidad histórica, en su matriz contextual, en su fundamentación vital y no solo como producto de la enfermedad".

Con base en lo planteado por Briñez y Muñoz (37), el abordaje de la visita familiar desde un enfoque comprensivo, se enmarca en el ámbito comunitario cuya aproximación se centra en los sujetos, sus acciones y los fenómenos sociales que determinan sus contextos, que van más allá de explicaciones causales, pues se reconoce de manera articulada y holística la presencia del todo en las partes ${ }^{\dagger^{* *}}$ (particularidades y procesos que por lo general no se perciben), incluyendo las familias como principales integradoras. Desde esta perspectiva, Vélez (49) expresa que le permite al profesional de la enfermería ampliar sus conexiones y relaciones con la comunidad, ingresar como un miembro más (entrar en la vida de las personas de una manera diferente) e interactuar en una dinámica de retroalimentación constante, en la que la realidad es concebida como un proceso permanente de interacción y construcción.

\footnotetext{
Aristóteles tenía claro que "el todo es más que la suma de las partes".
} 
En el marco del enfoque comprensivo se destaca la necesidad de un abordaje cultural, el cual, de acuerdo con Briñez y Muñoz (37) se muestra como una de las mejores alternativas a aplicar en el cuidado familiar al complementar la atención del modelo biomédico tradicional, dado que fomenta el respeto por la cultura y los saberes populares del sujeto de cuidado; además es coherente con el concepto de visita familiar, el cual a su vez concuerda con lo planteado por Cordero y Guevara (14) al referirse a ésta como práctica de aproximación a la realidad y a la dinámica familiar, lo que amerita la implementación de un modelo, teoría o enfoque disciplinar.

En cuanto a los enfoques teóricos analizados, aunque algunos no guardan relación con el enfoque comprensivo, existen algunos que servirían de guía para fomentar el pensamiento crítico, la toma de decisiones y diferenciar la enfermería de otras profesiones, según lo expresado por Parra-Giordano et al. (30), al visibilizar todas las acciones de cuidado familiar de una forma independiente y científica. Es así, como para la praxis desde un abordaje cultural, la teórica de la enfermería Madeleine Leininger, quien desarrolló el concepto de "enfermería transcultural", sustenta su postura a partir de la visión de interacción recíproca que consiste en ver a la persona como un ser holístico, biopsicosocial y cuya realidad depende del contexto; se propone dar respuesta a las necesidades planteadas y es coherente con lo propuesto por autores como Ruschel y Briñez $(37,45)$.

La práctica de la visita familiar con abordaje cultural resalta la utilización del lenguaje coloquial y la adaptación lingüista por parte del profesional $(36,37)$; se caracteriza por tener un diálogo de doble vía, por lo que autores como Monticelli et al. (50) y Rodríguez et al. (51) plantean que es importante superar la práctica impositiva del modelo biomédico ya que es unidireccional.

Por lo descrito y según la investigación documental realizada, llevar a cabo la visita familiar basada en la teoría de Leininger, permitirá que los profesionales de la enfermería se involucren activamente en el proceso de cuidado de los sujetos y la familia, a partir del conocimiento de sus saberes populares (45), lo que incluye sus creencias, valores, significados y modos de vida. En este sentido, un abordaje comprensivo y cultural, se traduce en un impacto positivo en la salud de la población en relación con sus microcontextos donde se resaltan las particularidades, se reconocen sus condiciones de vida y se incide en promover la equidad en salud (34), como también lo han descrito Vargas et al. (36) y Briñez et al. (37).

Se sugiere continuar investigando sobre el desarrollo de la visita familiar, donde se trascienda el enfoque habitual, que permita una mayor aproximación al cuidado de la salud personal, familiar y comunitaria; profundizar sobre el desarrollo teórico en la práctica, al reconocer la necesidad del cuidado cultural en la salud, incluso las posibilidades terapéuticas que pueden emerger desde el saber popular; promover en la academia la vinculación de escenarios formativos extramurales, participar en procesos investigativos desde ambientes cotidianos e incentivar la visita como medio para facilitar los procesos de enseñanza-aprendizaje.

\section{Conclusiones}

- Este estudio permitió identificar teorías y modelos de enfermería y de la psicología utilizados en el abordaje de la visita domiciliaria desde un enfoque familiar y cultural, lo cual evidencia que existe una amplia variedad y disponibilidad de éstas; no obstante, a pesar del interés que denotan las visitas domiciliarias para los equipos de salud y de manera particular para la enfermería, no hay consenso sobre la teoría, modelo o enfoque a aplicar.

- De acuerdo con esta investigación documental se propone la reconceptualización de la visita debido a la variedad de denominaciones que se le ha otorgado de manera indiscriminada, y considerar el concepto de tal forma que permita presentar abordajes alternativos, es por ello que se propone la denominación de visita familiar como:

- "Práctica de interacción con la comunidad, donde se busca un acercamiento al sujeto y a su familia en el contexto donde está inmerso, así como también, a las instituciones y otros entornos en donde se desarrolla(n), lo cual exige un esfuerzo de reflexión y acción por parte de los miembros de los equipos de Atención Primaria en Salud, en el sentido de indagar, comprender sus realidades sociales y realizar un acompañamiento en lo que han priorizado para ese momento de sus vidas, de modo que se definan prácticas de educación concretas y de cuidado con orientación cultural, ya sea de conservación, negociación o reorientación, lo que permitirá avanzar en su buen vivir". 
ISSN-PRINT

1794-9831

E-ISSN 2322-7028

Vol. 17 No. 1

Ene - Abr 2020

Cúcuta, Colombia
- Se hace relevante profundizar los postulados de la "teoría de la diversidad y universalidad: cuidados culturales" propuesta por Madeleine Leininger, para la implementación en la visita, en tanto, se evidenció que permite el logro de los objetivos que se persiguen por medio de la visita familiar, según la reconceptualización propuesta.

\section{Agradecimientos}

Las autoras agradecen a la Vicerrectoría de Investigaciones y Posgrados de la Universidad de Caldas por el financiamiento de la investigación. También a los
Grupos "Promoción de la Salud y Prevención de la Enfermedad" y "CUSAVI" por incentivar, por medio de las reuniones, la reflexividad constante del ejercicio profesional en relación con las demandas del contexto y la necesidad, cada vez mayor, de responder con calidad y pertinencia en el ámbito extramural, a través de la participación de la enfermería en equipos interprofesionales que implementan la estrategia de APS.

\section{Conflictos de intereses}

Las autoras declaran no tener ningún tipo de conflicto de intereses.

\section{Referencias bibliográficas}

1. World Health Organization and United Nations Children's Fund (UNICEF). Conferencia Mundial sobre Atención Primaria de Salud: desde Alma-Ata hacia la cobertura sanitaria universal y los Objetivos de Desarrollo Sostenible. [Internet]. Astaná (Kazajstán); 2018 [citado 2019 Feb 8]. Disponible en: https:/www.who.int/docs/default-source/primary-health/declaration/gcphc-declaration-sp.pdf

2. Harzheim E, Casado Vicente V, Bonal Pitz P, Oswaldo Cruz F, Giovanella L, Rivas A, et al. La formación de profesionales de salud para la APS y Salud Familiar y Comunitaria en América Latina y Europa [Internet]. Porto Alegre; 2008 [citado 2019 Ene 24]. Disponible en: https://www.argentina. gob.ar/sites/default/files/harzheim.casadovicente-formacion_profesional_para la aps.pdf

3. Coppa D, Schneidereith T, Farina CL. Simulated Home-Based Health Care Scenarios for Nurse Practitioner Students. Clin Simul Nurs. 2019; 26:38-43.

4. González Pascual JL, Cuesta Rubio N, Sanz Pozo B, González Sanz P, López Romero A, Muñoz Balsa MJ, et al. Educación interprofesional a través de la atención domiciliaria: experiencia tras 2 años de implementación en los grados de Medicina y Enfermería de la Universidad Europea de Madrid. Educ Med. 2019;20(1):2-7.

5. Vélez Álvarez C, Jaramillo Ángel CP, Giraldo Osorio A. Docencia-servicio: responsabilidad social en la formación del talento humano en salud en Colombia. Educ Med. 2018;19(S2):179-86.

6. Kotliarenco M, Gómez E, Muñoz M, Aracena M. Características, efectividad y desafíos de la visita domiciliaria en programas de intervención temprana. Rev Salud Pública. 2010;12(2):184-96.

7. Ministerio de Salud y Protección Social. Resolución número 00412 de 2000. Por la cual se establecen las actividades, procedimientos e intervenciones de demanda inducida y obligatorio cumplimiento y se adoptan las normas técnicas y guías de atención para el desarrollo de las acciones de protección específica y detección temprana y la atención de enfermedades de interés en salud pública. Bogotá D.C; 2000.

8. Zuliani Arango LA, Villegas Peña ME, Galindo Cárdenas LA, Kambourova M. Visita domiciliaria familiar: estrategia didáctica para la formación integral del personal médico. Rev Latinoam Ciencias Soc Niñez y Juv [Internet]. 2015 [citado 2019 Feb 5]; 3(2): 851-63. Disponible en: http://www.scielo. org.co/pdf/rlcs/v13n2/v13n2a22.pdf

9. Olaya Cuadros BL, Gaviria Noreña DL. Cuidado de la salud de la familia. Rev cienc cuidad. 2017; 14(1):79-94.

10. Díaz Fernández JK, Velásquez Gutierrez VF, López Díaz AL. Efecto programa de salud domiciliaria 
en personas ancianas de grupos culturalmente diversos. Rev cienc cuidad. 2018;15(2):79-90.

11. Ministerio de Salud y Protección Social. La Política de Atención Integral en Salud: "hacia un nuevo modelo de atención integral en salud" [Internet]. Ministerio de Salud y Protección Social. 2016. Disponible en: https://www.minsalud.gov.co/Documentos $\% 20 \mathrm{y} \% 20 \mathrm{Publicaciones} / \mathrm{Pol} \% \mathrm{C} 3 \%$ ADtica $\% 20$ de\%20Atenci\%C3\%B3n\%20Integral\%20en\%20Salud.pdf

12. Coffey A, Atkinson P. Encontrar el sentido a los datos cualitativos. Estrategias complementarias de investigación. 1era ed. Universidad de Antioquia, editor. Medellín, Antioquia; 2003. 132 p.

13. Osses Bustingorry S, Sánchez Tapia I, Ibáñez Mansilla FM. Investigación cualitativa en educación: hacia la generacion de teoría a través del proceso analítico. Estud pedagógicos [Internet]. 2009 [citado 2019 Feb 11]; 32(1): 19-33. Disponible en: http://www.scielo.cl/scielo.php?script=sci_arttext\&pid=S071807052006000100007\&lng=en\&nrm $=$ iso\&tlng $=$ en

14. Cordero Solís K, Guevara Francesa G. Programa de visita domiciliaria del sistema de salud costarricense: lineamientos para su mejora. Enferm Actual Costa Rica [Internet]. 2015 [citado 2019 Feb 11]; 2015; 29:1-18. Disponible en: http://revistas.ucr.ac.cr/index.php/enfermeria/article/view/19671

15. Brugerolles ME, Mena $\mathrm{C}$, Dois A. Beneficio de la visita domiciliaria para los adultos mayores. Horiz Enferm [Internet]. 2008 [citado 2019 Feb 11]; 19(2):55-64. Disponible en: http://horizonteenfermeria. uc.cl/images/pdf/19-2/benficio visita domiciliaria.pdf

16. Rodrigues Melo TM, Vale Oliveira do LM, Leitão Rodrigues RA, Silva Oliveira da RM, Rocha Santiago da S, Pedrosa dos Santos JI. A visita domiciliar do enfermeiro à puérpera e ao recém-nascido. NOVAFAPI [Internet]. 2011 [citado 2019 En 28]; 4(2):21-6. Disponible en: https://revistainterdisciplinar.uninovafapi.edu.br/revistainterdisciplinar/v4n2/pesquisa/p3_v4n2.pdf

17. Machado dos Santos E, Gomes Morais SH. A visita domiciliar na estratégia saúde da família: percepção de enfermeiros. Cogitare Enferm. 2011;16(3):492-7.

18. Sivalli Campos CM, Buffette Silva BR, Forlin DC, Trapé CA, de Oliveira Lopes I. Práticas emancipatórias de enfermeiros na Atenção Básica à Saúde: a visita domiciliar como instrumento de reconhecimento de necessidades de saúde. Rev da Esc Enferm da USP [Internet]. 2014 [citado 2019 En 28]; 48(spe):119-25. Disponible en: http://www.scielo.br/scielo.php?script=sci_arttext\&pid=S008062342014000700119\&lng=en\&tlng $=$ en

19. Santos Nascimento M, Alves do Nascimento MA. Prática da enfermeira no Programa de Saúde da Família: a interface da vigilância da saúde versus as ações programáticas em saúde. Ciência \&amp; Saúde Coletiva. 2005; 10(2):333-45.

20. Salgueiro Nascimento J, Crispim Costa L de M, dos Santos RM, Santos dos Anjos D. Visitas domiciliares como estratégias de promoção da saúde pela enfermagem. Rev Bras em promoção da Saúde [revista en Internet]. 2013 [citado 2019 Ene 29]; 26(4): 513-22. Disponible en: https://periodicos. unifor.br/RBPS/article/view/3116

21. Aston M, Price S, Etowa J, Vukic A, Young L, Hart C, et al. The power of relationships: exploring how public health nurses support mothers and families during postpartum home visits. J Fam Nurs. 2015;21(1):11-34.

22. Sawyer MG, Pfeiffer S, Sawyer A, Bowerin K, Jeffs D, Lynch J. Effectiveness of nurse home visiting for families in rural South Australia. J Paediatr Child Health. 2014; 50(12):1013-22.

23. Dawley K, Loch J, Bindrich I. The nurse - family pathernship. American Journal of Nursing. 2007; 107(11):60-7.

24. Mejdoubi J, van den Heijkan S, van Leerdam FJ, Heymans MW, Crijnen A, Crijnen RA. The effect of VoorZorg, the dutch nurse-family partnership, on child maltreatment and development: A randomized controlled trial. PLoS One [Internet]. 2015; 10(4):1-14. Disponible en: http://dx.doi.org/10.1371/ journal.pone. 0120182

25. Monsen KA, Radosevich DM, Kerr MJ, Fulkerson JA. Public health nurses tailor interventions for 
ISSN-PRINT

1794-9831

E-ISSN 2322-7028

Vol. 17 No. 1

Ene - Abr 2020

Cúcuta, Colombia

families at risk. Public Health Nurs. 2011; 28(2):119-28.

26. Monsen K, Sanders A, Yu D, Radosevich D, Geppert J. Family home visiting outcomes for mothers with and without intellectual disabilities. J Intellect Disabil Res. 2011;55(5):484-99.

27. Figueras Aloy J, Esqué Ruiz MT, Arroyo Gili L, Bella Rodríguez J, Álvarez Miró R, Carbonell Estrany $\mathrm{X}$. Evolución de la atención a domicilio para niños prematuros y de bajo peso de nacimiento desde 2002 hasta 2014. An Pediatr (Barc). 2016;85(3):134-41.

28. Rebollo PE, Martín TB, Téllez de la Vegac E. Visita domiciliaria al recién nacido y a la puérpera. An Sist Sanit Navar. 2002;25(SUPPL. 2):7-16.

29. Alves Kebian LV, Acioli S. Visita domiciliar: espaço de práticas de cuidado do enfermeiro e do agente comunitário de saúde. Rev Enferm [revista en Internet]. 2011 [citado 2019 En 28];19(3): 403-9. Disponible en: http://pesquisa.bvs.br/brasil/resource/pt/lil-618864

30. Parra Giordano D, González Molina D, Pinto Galleguillos D. Proceso de enfermería en un estudio de familia de persona adulta mayor en atención primaria de salud. Enfermería Univ [Internet]. 2017 [citado 2019 En 28]; 14(1):67-75. Disponible en: http://linkinghub.elsevier.com/retrieve/pii/ $\underline{\mathrm{S} 1665706316300665}$

31. Parra Giordano D. Revolución científica de la salud familiar en Chile. Rev Enfermería Actual en Costa Rica [revista en Internet]. 2016 [citado 2019 Ene 28]; 31:1-16. Disponible en: https://dialnet. unirioja.es/servlet/articulo?codigo $=5571649$

32. Melnick AL, Rdesinski RE, Creach ED, Choi D, Harvey SM. The Influence of Nurse Home Visits, Including Provision of 3 Months of Contraceptives and Contraceptive Counseling, on Perceived Barriers to Contraceptive Use and Contraceptive Use Self-Efficacy. Women's Heal Issues [Internet]. 2008 [citado 2019 Ene 28]; 18(6):471-81. Disponible en: https://linkinghub.elsevier.com/retrieve/pii/ $\underline{\mathrm{S} 1049386708001035}$

33. Nicolaides Bouman A, Van Rossum E, Habets H, Kempen G, Knipschild P. Home visiting programme for older people with health problems: Process evaluation. J Adv Nurs. 2007; 58(5):425-35.

34. Abbott LS, Elliott LT. Eliminating health disparities through action on the social determinants of health: a systematic review of home visiting in the United States, 2005-2015. Public Health Nurs. 2017; 34(1):2-30.

35. Corrales Nevado D, Palomo Cobos L. La importancia de la longitudinalidad, integralidad, coordinación y continuidad de los cuidados domiciliarios efectuados por enfermería. Enferm Clin. 2014; 24(1):51-8.

36. Vargas Acevedo CA, Alzate Posada ML, López Díaz L, Velásquez VF. Dolor en personas ancianas con dependencia: programa de visita domiciliaria adaptado culturalmente a población rural. Rev Salud pública. 2017; 19(4):519-26.

37. Briñez Ariza KJ, Muñoz de Rodriguez L. Experiencias de cuidado cultural en personas con diabetes y el contexto familiar, con enfoque Leininger. Cult los Cuid. 2016; 20(45):81-90.

38. Loboa Rodríguez NJ, León Saavedra PE. Conocimientos y actitudes de enfermería sobre promoción de la salud: una mirada de región. IEID [Internet]. 2018; 20(1). Available from: https://revistas.javeriana.edu.co/index.php/imagenydesarrollo/article/view/16716

39. Depaux R, Campodónico L, Ringeling I, Segovia I. En el camino a centro de salud familiar [Internet]. Ministerio de Salud. Santiago, Chile; 2008 [citado 2019 Feb 23]. 133 p. Disponible en: http://buenaspracticasaps.cl/wp-content/uploads/2014/07/MINSAL-2008-En-el-camino-a-Centro-de-SaludFamiliar.pdf

40. Briceño T. El paradigma científico y su fundamento en la obra de Thomas Kunhn. Tiempo y Espac [Internet]. 2009 [citado 2019 Mar 1]; 19(52): 285-97. Disponible en: https://dialnet.unirioja.es/servlet/articulo? codigo $=3579139$

41. Mackinnon CJ. Applying feminist, multicultural, and social justice theory to diverse women who 
function as caregivers in end-of-life and palliative home care. Palliat Support Care. 2009;7(4):501-12.

42. Flynn L, Budd M, Modelski J. Enhancing Resource Utilization Among Pregnant Adolescents. Public Health Nurs [Internet]. 2008 [citado 2019 Ene 28]; 25(2):140-8. Disponible en: http://doi.wiley. com/10.1111/j.1525-1446.2008.00690.x

43. Medeiros de Oliveira JM, Cunha De Araújo JP, Ferreira de Lima HC, Santos de Lucena P, Silva de Farias PH, Paiva de Menezes RM. O cuidado de enfermagem na visita domiciliar gerontológica: uma perspectiva humanística. Ciência, Cuid e Saúde [Internet]. 2013 [citado 2019 Ene 28]; 12(1):170-6. Disponible en: http://periodicos.uem.br/ojs/index.php/CiencCuidSaude/article/view/16672

44. Mesquita Melo E, Lop es M de O, Carvalho Fernandes A, Lima T, Barbosa I. Teorías de enfermería: importancia de la correcta aplicación de los conceptos. Enfermería Glob [Internet]. 2009 [citado 2019 Mar 1]; (17):1-10. Disponible en: http://scielo.isciii.es/scielo.php?script=sci_arttext\&pid=S169561412009000300017\&lng=en\&nrm=iso\&tlng=en

45. Ruschel KB, Azzolin KO, Rabelo ER. Visita domiciliar para pacientes con insuficiencia cardíaca: una reflexión sobre la luz de la teoria de Leininger. Online braz j nurs [Internet]. 2012 [citado 2019 Ene 29];11(3):883-92. Disponible en: https://docplayer.es/22919599-Visita-domiciliar-para-pacientescon-insuficiencia-cardiaca-una-reflexion-sobre-la-luz-de-la-teoria-de-leininger.html

46. Rodríguez S, Cárdenas M, Pacheco AL, Ramírez M, Ferro N, Alvarado E. Reflexión teórica sobre el arte del cuidado. Enfermería Univ [Internet]. 2017 [citado 2019 Ene 29]; 14(3):191-8. Disponible en: http://linkinghub.elsevier.com/retrieve/pii/S1665706317300404

47. Xiong S, Degroote N, Byington H, Harder J, Kaminski K, Haglund K. Engaging in Culturally Informed Nursing Care With Hmong Children and Their Families. J Pediatr Nurs. 2016; 31(1):102-6.

48. Granda E. ¿A qué llamamos salud colectiva, hoy?. Rev Cuba Salud Pública [Internet]. 2008 [citado 2019 Feb 23]; 30(2):1-15. Disponible en: https://www.redalyc.org/comocitar.oa?id=21430209

49. Vélez Restrepo OL. Reconfigurando El Trabajo Social. Perspectivas y Tendencias Contemporáneas. Editorial Espacio, editor. Buenos Aires; 2003. 81 p.

50. Monticelli M, Boehs AE, Guesser JC, Gehrmann T, Paiva K. Perfil de dissertações que utilizam a teoria de Leininger vinculadas a um programa de mestrado em enfermagem do sul do país. Ciência Cuid e Saúde. 2008; 7(4): 447-53.

51. Rodríguez Villamil N, Valencia González A, Díaz Mosquera P. Promoción de la salud y sistema de salud colombiano: reflexiones sobre la formación de profesionales. Hacia Promoc. Salud. [revista en Internet]. 2013 [citado 2019 Feb 5];18(1):26-40. Disponible en: https://www.redalyc.org/articulo. oa?id $=309126802003$ 